\title{
Counterfactuals and Explanatory Pluralism
}

\author{
Kareem Khalifa Gabriel Doble Jared Millson
}

\begin{abstract}
Recent literature on noncausal explanation raises the question as to whether explanatory monism, the thesis that all explanations submit to the same analysis, is true. The leading monist proposal holds that all explanations support change-relating counterfactuals. We provide several objections to this monist position.
\end{abstract}

\section{Introduction}

2 CR-Monism's Three Problems

3 Dependency and Monism: Unhappy Together

4 Another Challenge: Counterfactual Incidentalism

4.1 High-grade necessity

4.2 Unity in diversity

5 Conclusion

\section{Introduction}

Beginning in the late 1980s, causal accounts of explanation came to the fore in the philosophy of science (Cartwright [1989]; Salmon [1984]; Strevens [2008]; Woodward [2003]). By the mid-2000s, several philosophers cited detailed examples of noncausal explanations from a 
wide variety of scientific fields. ${ }^{1}$ Among the central debates in this burgeoning literature is whether the existence of noncausal explanations suggests a pluralistic or monistic account of explanation. Pluralists claim that causal and noncausal explanations are different kinds of explanations that elude a single analysis. Monists, by contrast, claim that causal and noncausal explanations exhibit a common characteristic that makes them explanatory. ${ }^{2}$

A growing consensus holds that change-relating (CR) counterfactuals are the unifying characteristic of both causal and noncausal explanations (Bokulich [2011]; French and Saatsi [2018]; Jansson and Saatsi [2017]; Pexton [2014]; Reutlinger [2016]; Rice [2015]; Saatsi [2018]; Saatsi and Pexton [2013]; Woodward [2003]). On this view, all explanations provide answers to 'what-if-things-had-been-different (w-) questions,' to use Woodward's memorable catchphrase. Call this position 'CR-monism.'

In this paper, we argue that CR-monism faces several challenges that have not been addressed. Section 2 describes CR-monism and introduces three problems with it. Section 3 shows that solutions to two of these problems are at cross-purposes. Whether CR-monists aim to provide both necessary and sufficient conditions for explanations underlies this tension. Section 4 argues that the latter aim should be abandoned, but that CR-monists still must rule out cases where explanations only happen to support change-relating counterfactuals but do not fulfill their main functions by virtue of these counterfactuals. We then show that even this more modest form of CR-monism faces problems. Thus, CR-monism's core claims are insufficient for explanation, and are unnecessary for executing several functions that explanations serve. Section 5 then concludes with broader lessons about the monist-pluralist debate.

${ }^{1}$ We review some of the examples below. See Lange ([2016]) and Reutlinger ([2017a]) for more comprehensive reviews of noncausal explanation. We also restrict our discussion to explanations of empirical phenomena, bracketing explanations in pure mathematics, moral theory, or other domains that are not (obviously) empirical.

${ }^{2}$ In later work, we hope to engage the monist proposal that all explanations are causal, advanced by, Skow ([2013]), for example. 


\section{CR-Monism's Three Problems}

Precise formulations of CR-monism are few and far between. Reutlinger ([2016]) is an exception:

[T] he relationship between the explanans [consisting of generalizations $G_{1}, \ldots, G_{m}$ and auxiliary statements $\left.S_{1}, \ldots, S_{n}\right]$ and the explanandum $[E]$ is explanatory iff the following conditions are all satisfied:

1. Veridicality condition: $G_{1}, \ldots, G_{m}, S_{1}, \ldots, S_{n}$ and $E$ are (approximately) true.

2. Implication condition: $G_{1}, \ldots, G_{m}$, and $S_{1}, \ldots, S_{n}$ logically entail $E$ or a conditional probability $P\left(E \mid S_{1}, \ldots, S_{n}\right)$-where the conditional probability need not be 'high', in contrast to Hempel's covering law account.

3. Dependency condition: $G_{1}, \ldots, G_{m}$ support at least one counterfactual of the following form: had $S_{1}, \ldots, S_{n}$ been different than they actually are (in at least one way deemed possible in the light of the generalizations), then $E$ or the conditional probability of $E$ would have been different as well. (Reutlinger [2016], pp. 737)

On this view, all explanations answer w-questions (per the dependency condition.) $)^{3}$

Moreover, this implies that all sets of propositions that also satisfy the veridicality and implication conditions are explanatory.

Reutlinger's proposal faces three challenges that all CR-monists should address. First, some CR-monists argue that explanations relying on idealizations belie the veridicality condition (Bokulich [2011]; Pexton [2014]; Rice [2015]). We will not discuss this in what follows, though we think that a broader notion of what counts as representational success with respect to explanations readily addresses it.

Second, the dependency condition appears too weak. Consider, for instance, Moore's law, eponymously dubbed after the chairman emeritus of Intel:

$($ Transistors per computer chip $)=2^{(\text {year-1975)/1.5 }}$

Coupled with values for different years, this 'law' satisfies Reutlinger's three conditions, yet it yields no explanation. To see this, consider the CR-counterfactuals that Moore's law

${ }^{3}$ We are puzzled that Reutlinger ([2016], pp. 735) offers necessary and sufficient conditions for any explanatory relation, yet also claims 'not to want to argue [...] that the counterfactual theory captures all kinds of noncausal explanations.' Is this simply a reflection of what he does not want to argue or does it further reflect his beliefs? At any rate, we want to argue that the counterfactual theory does not capture all kinds of noncausal explanations! 
supports, for example, had the year been 1975, then there would have been only one transistor per chip. This fails to be explanatory because the year merely correlates with the real explanatory factor behind the growth in computing power. Thus, the dependency condition is clearly the problem. Let 'dependency problems' denote non-explanations that support change-relating counterfactuals.

Dependency problems threaten many CR-monists. For instance, Saatsi and Pexton ([2013]) clearly invite this worry:

All that matters for explanatory power $[\ldots]$ is there being objective facts about the relevant counterfactual circumstances and having epistemic access to these facts. (Saatsi and Pexton [2013], pp. 631)

Moreover, several CR-monists are vague enough on this point to occasion this concern. ${ }^{4}$ Hence, CR-monists need to delineate explanatory and non-explanatory counterfactuals.

Solving dependency problems requires a more demanding successor to Reutlinger's dependency condition. Yet, it must neither be so demanding that acceptable explanations fall outside of CR-monism's tent, nor address these issues by allowing different explanations to avoid dependency problems in substantially different ways, as these would amount to pluralist positions. Hence, we dub CR-monists' third and final challenge 'monism problems.'

\section{Dependency and Monism: Unhappy Together}

As just noted, CR-monists face a possible trade-off in solving dependency and monism problems. ${ }^{5}$ The former require strengthening the dependency condition; the latter, weakening it. To that end, we rehearse the difficulties involved in this 'dependency-monism dilemma' (DMD).

We can see CR-monists as falling into two camps: criterial and quasi-interventionist. Both succumb to the DMD. According to criterial CR-monists, only causal explanations must satisfy all members in a list of criteria—such as asymmetry and distinctness of explanatory

\footnotetext{
${ }^{4}$ In fairness, many are more interested in showing that certain scientific explanations fit the CR-monist template than showing why non-explanations do not.

${ }^{5}$ Pincock ([Forthcoming]) raises a similar dilemma. Our argument differs from his in delving more deeply into possible CR-monist replies.
} 
relata—while noncausal explanations satisfy only a subset (Craver and Bechtel [2007], pp. 551-555; Reutlinger [2016], pp. 737-738; Rice [2015], pp. 607). However, criterial approaches face several difficulties. First, none of the current proposals indicate which of these criteria disqualify pseudo-explanations while at the same allowing for noncausal explanations. For instance, transistor capacity and the year are metaphysically distinct, so pseudo-explanations satisfy some of these criteria. Hence, it is unclear how criterial CR-monists solve dependency problems.

Second, according to criterial CR-monism, the veridicality, implication, and dependency conditions would only be necessary for all explanations, but there would be one or more sets of additional criteria that are sufficient for noncausal explanations but insufficient for causal explanations. Hence, criterial approaches also face monism problems.

In contrast to criterialists, quasi-interventionist CR-monists urge a more modest departure from causal theories of explanation (Jansson and Saatsi [2017]; Pexton [2014]). The guiding idea is this: causal explanations are the best-understood species of CR explanations, and have abundant resources for avoiding dependency problems. So, CR-monism can be developed by making the most conservative changes to a well-developed theory of causal explanation that are liable to capitalize on these resources, while still solving the monism problem by also covering all noncausal explanations.

However, previous quasi-interventionists have merely pointed to this idea without much detail. Since we find this idea highly suggestive, we will unpack it on their behalf, by taking Woodward's interventionist account of causal explanation as our starting point. We will then show that the most plausible ways of formulating quasi-interventionism cannot escape the DMD.

According to Woodward, causal explanations support counterfactuals of the form, if a cause $X$ were to change under an intervention, then its effect $Y$ would also change. Woodward ([2003]) defines interventions thusly:

(IN) $I$ 's assuming some value $I=z_{i}$, is an intervention on $X$ with respect to $Y$ if and only if

a. $I$ is an intervention variable for $X$ with respect to $Y$ and

b. $I=z_{i}$ is an actual cause of the value taken by $X$. (Woodward [2003], pp. 98) 
IN.b's details figure only derivatively in what follows, so we leave them implicit hereafter.

Noncausal explanations must bypass one of Woodward's requirements on IN.a, namely, his account of an intervention variable:

(IV) Some variable $I$ is an intervention variable for $X$ with respect to $Y$ if and only if:

I1. $I$ causes $X^{6}$

I2. $I$ acts as a switch for all the other variables that cause $X$. That is, certain values of $I$ are such that when $I$ attains those values, $X$ ceases to depend on the values of other variables $[V]$ that cause $X$ and instead depends only on the value taken by $I$.

I3. Any directed path from $I$ to $Y$ goes through $X$. That is, $I$ does not directly cause $Y$ and is not a cause of any causes $[W]$ of $Y$ that are distinct from $X$ except, of course, for those causes of $Y$, if any, that are built into the $I-X-Y$ connection itself; that is, except for (a) any causes of $Y$ that are effects of $X$ (namely, variables that are causally between $X$ and $Y$ ) and (b) any causes of $Y$ that are between $I$ and $X$ and have no effect on $Y$ independently of $X$.

I4. $I$ is (statistically) independent of any variable $Z$ that causes $Y$ and that is on a directed path that does not go through $X$. (Woodward [2003], pp. 98)

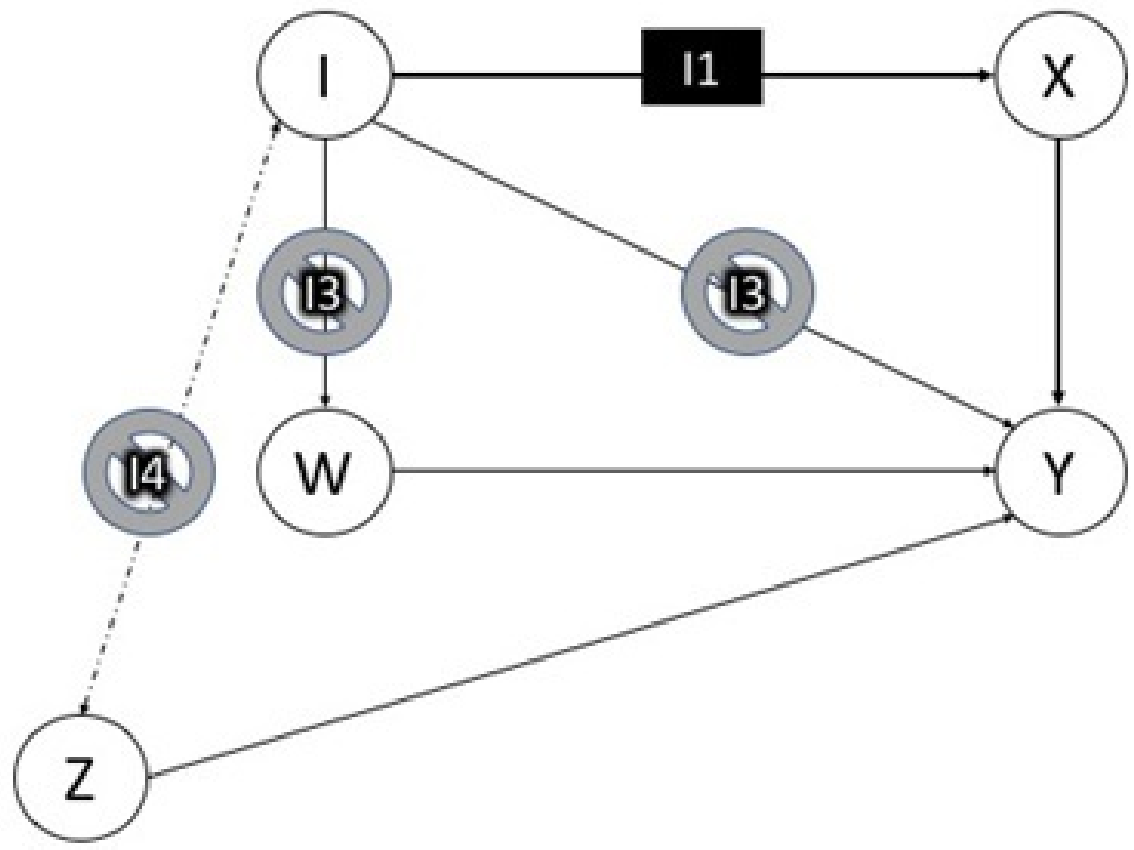

Figure 1: Woodward's account of interventions. The solid arrows indicate causal relations; the dotted line, a correlation; the black box, the requirement I1; and the gray, encircled strikethroughs, the restrictions I2-I4 discussed above.

As Figure 1 illustrates, I1-I4 specify an ideal experimental intervention: experimental

${ }^{6}$ Woodward ([2003], pp. 104-107) argues that the apparent circularity of defining causes in terms of interventions is not vicious. 
interventions that satisfied these conditions would show that only $X$ (as opposed to $V, W$, or $Z$ ) is the cause of $Y$. This tells us why no causal explanation can rely on Moore's law; there is no way of changing transistor capacity simply by intervening on the year; such a change will always go through other variables that cause changes in transistor capacity and are correlated with the year, thereby violating either I 3 or I4. ${ }^{7}$

The most conservative change to (IV) that allows quasi-interventionism to cover noncausal explanations would simply reinterpret the arrows on the $I-X-Y$ path as noncausal counterfactual dependency relations. In effect, this simply means replacing I1 with:

\section{Q1. $X$ counterfactually depends on $I$,}

and assuming that when $\mathrm{Q} 1$ is conjoined with $\mathrm{I} 2-\mathrm{I} 4, Y$ counterfactually depends on $X$, and $I$ is a quasi-intervention on $X$ with respect to $Y$. This clearly solves the aforementioned dependency problem, for it shares precisely those conditions that blocked Moore's law from being explanatory (I3 and I4.) Indeed, it would appear to be able to steal many pages from Woodward's book. Furthermore, if this quasi-interventionist approach captured every kind of explanation, causes would just be a limiting case: quite plausibly, if $X$ causes $Y$, then $Y$ counterfactually depends on $X$. So, quasi-interventionism has many initial attractions.

However, it still faces monism problems. Specifically, a very prominent class of noncausal explanation-constitutive explanations_-violates I3 (Craver and Bechtel [2007]; Ylikoski [2013]). ${ }^{8}$ In constitutive explanations, a system's components and organization explain some of its properties. Consider Ylikoski’s ([2013], pp. 231) account: 'System $S$ has causal capacity $k$ in circumstances $T$, due to S's components $S_{1} \ldots S_{n}$ and their organization $O$.' Constitutive explananda - a system's 'causal capacities' - are its dispositions under certain enabling and triggering conditions. For example, suppose that we ask why a group has a particular kind of problem-solving capacity (Ylikoski [2014], pp. 124). Causal explanations cite the causal history of the group and its members, but constitutive explanations cite

\footnotetext{
${ }^{7}$ In I3, correlations result from the intervention variable $I$ being a common cause either of $W$ and $X$ or of $X$ and $Y$.

${ }^{8}$ While we focus on constitutive explanations, we consider them part of a broader family of 'tangled' explanations—-reviewed by Kostić ([2016])—that violate I3.
} 
properties of the group's members and their organization, such as the members' intelligence and the social norms characterizing their interactions.

While causal relata are metaphysically independent entities, constitutive relata 'are not independent existences, so one cannot think of an intervention on the basis that would not also be an intervention on the system' (Ylikoski [2013], pp. 284). Thus, while removing the group's smartest member would change the explanans, group membership, it would also be a direct change to the problem-solving capacities of the group, i.e. the explanandum. This violates I3. Yet, explaining the group's problem-solving capacities in terms of what its members can do is perfectly acceptable. Hence, if $\mathrm{I} 3$ is a requirement on quasi-interventions, then constitutive explanations pose monism problems.

In response, quasi-interventionists might be tempted to relax $\mathrm{I} 3$ as a requirement on quasi-interventions. However, this raises dependency problems. Note that I3 blocks interventions from being common causes (Woodward [2003], pp. 100-103). Consider a putative intervention variable, say hot weather $(I)$, that is a common cause of two other variables, say ice cream consumption $(X)$ and murder $(Y)$. I3 is not satisfied, so Woodward's IV blocks the absurd claim that ice cream consumption explains murder. It is unclear how the quasi-interventionist proposal under consideration delivers the same result. Thus, even when the veridicality and implication conditions are conjoined to quasi-interventionists' more detailed specifications of the dependency condition, the result is (at best) necessary for explanation, but not sufficient.

\section{Another Challenge: Counterfactual Incidentalism}

From the preceding, we conclude that CR-monists have not resolved the dependency-monism dilemma. For the sake of argument, we consider a more modest brand of CR-monism that permits some dependency problems. Intuitively, CR-monists ought to place greater weight on identifying necessary conditions than on identifying sufficient conditions, as 'CR-monism' seems to mislabel any position that allows some non-change-relating explanations.

Conversely, showing that all explanations satisfy a set of nontrivial conditions seems a significant form of monism, even if non-explanations also satisfy those conditions. Thus, 
dependency problems, but not monism problems, are tolerable.

To that end, let minimal CR-monism assert that generalizations $G_{1}, \ldots, G_{m}$ and auxiliary statements $S_{1}, \ldots, S_{n}$ explain $E$ only if the veridicality, implication, and dependency conditions are satisfied. ${ }^{9}$ For all that we will argue, minimal CR-monism may well be true. However, it should provide cold comfort to its devotees. The reason why concerns a challenge that Lange ([2016]) has pitched to causal monists, but that can be transposed to CR-monists (minimal or otherwise.) We call Lange's original challenge 'causal incidentalism:'

That a distinctively mathematical explanation happens to cite facts about the explanandum's causes does not mean that it works by virtue of describing the explanandum's causes. (Lange [2016], pp. 20)

Our goal is to generalize this claim in two ways. First, and most obviously, at least some noncausal explanations satisfy minimal CR-monism's requirements. Hence, we should replace Lange's references to causes with references to counterfactual dependence. Second, we do not restrict this claim to distinctively mathematical explanations. Combined, this suggests:

Counterfactual Incidentalism: Some explanations happen to satisfy the dependency condition, but do not work by virtue of supporting counterfactuals that relate changes in their explanantia to changes in their explananda.

We claim that even if all explanations satisfy the dependency condition, some do so incidentally. A crucial task, then, is to articulate what it means for an explanation "not to work by virtue of' trafficking in change-relating counterfactuals. Let us consider a simple example of this locution: cars do not work by virtue of being designed by members of Homo sapiens. Why is this? Because even if some other (sufficiently intelligent) species designed a car, it would still serve its chief function of transporting people. By parity of reasoning, we claim that some explanations would execute their chief functions even if those explanations did not support change-relating counterfactuals. In such cases, the explanations do not work by virtue of satisfying the dependency condition.

If some explanations are only incidentally change-relating in this way, then even if minimal

${ }^{9}$ Recall that Reutlinger ([2016]) took these three conditions to be necessary and sufficient for explanation. 
CR-monism is true, it is not clear why it is worth fighting for. For certain explanations, the dependency condition would, for all practical purposes, be irrelevant. At best, the veridicality and implication conditions would not be incidental, but this would mean that the one thing that makes all explanations work are derivations of the explanandum from accurate generalizations and auxiliary statements. The resulting 'monism' amounts to little more than watered-down Hempelianism.

Thus, we claim that counterfactual incidentalism threatens any interesting brand of CR-monism. Explanations appear to serve many functions: showing that the explanandum was to be nomically expected, fitting the explanandum within the causal structure of the world, unifying scientific knowledge, providing information potentially exploitable for interventions, filling knowledge-gaps by answering why-questions, and showing that an explanandum had to have happened. ${ }^{10}$ To avoid incidentalist challenges, CR-monists must show that all of these functions require satisfaction of the dependency condition, or else deny that they are explanatory functions. These tasks are nontrivial, and CR-monists have, at best, only gestured toward their completion.

In the examples below, we identify two explanatory functions that can sometimes be realized even if the dependency condition were flouted: conferring 'high-grade' necessity upon explananda (Section 4.1) and finding unity in apparent diversity (Section 4.2). In both cases, the change-relating counterfactuals they support are, at best, incidental.

\subsection{High-grade necessity}

As Lange ([2016], pp. 12) observes, some explanations work 'by showing the explanandum to be necessary in a stronger sense than any causal explanation could.' Some CR-monists counter that even though this is true, those explanations do not show the explanandum to be necessary in a stronger sense than any change-relating explanation could (Jansson and Saatsi [2017];

\footnotetext{
${ }^{10}$ In a similar vein, Hochstein ([2017]) argues that explanations serve multiple scientific goals. Our view is consistent with (but does not entail) Hochstein's further claim that multiple models are needed to provide a good scientific explanation. Conversely, Hochstein's view is consistent with (but does not entail) our critique of CR-monism.
} 
Reutlinger [2016]). Counterfactual incidentalism renders this CR-monist maneuver moot: even if they satisfy the dependency condition, some explanations show an explanandum to be necessary in a strong sense but not by virtue of supporting change-relating counterfactuals. ${ }^{11}$ After presenting this argument, we anticipate and rebut several CR-monist objections. ${ }^{12}$ Consider the following example discussed by Lange ([2016]). Why, at every moment, do there exist antipodal points on the equator having the same temperature? The answer to this question does not invoke local meteorological causes of each location's temperature, which would only explain why each antipodal point has the temperature it does, but not why there must be two antipodal points with the same temperature. For that, we must appeal to a mathematical fact, the intermediate value theorem. This theorem states that for any real-valued, continuous function $f$, and real number $u$ between $f(a)$ and $f(b)$, there exists a value $c \in[a, b]$ such that $f(c)=u$. Surprisingly, this fact about real-valued, continuous functions tells us something about equatorial temperatures.

To explain why a pair of antipodal points on the equator must have the same temperature, consider the function, $D(x)$, which is simply the difference between the temperature at equatorial point $x$ and at its antipode, $x^{\prime}$. Specifically, if $T(x)$ represents the temperature at $x$, then $D(x)=T(x)-T\left(x^{\prime}\right)$. Since the temperature on the equator is a real-valued, continuous function, so is the difference between the temperature of any equatorial point and its antipode. Now, consider any point $x$ on the equator that is warmer than its antipode $x^{\prime}$. Since $T(x)-T\left(x^{\prime}\right)$ will be a positive number, $D(x)>0$. Since $T\left(x^{\prime}\right)-T(x)$ will be a negative

\footnotetext{
${ }^{11}$ More liberal (non-CR) monists might unify all explanations through their capacity to
} answer w-questions. They would differ from CR-monists in claiming that some non-change-relating counterfactuals answer w-questions. The most promising candidates on this front are counterfactuals describing how the explanandum would remain constant under many changes to the explanandum. Section 4.2 discusses these 'stability-exhibiting counterfactuals' in detail, and implies that this liberal monism is merely pluralism in disguise.

${ }^{12}$ Space prohibits discussion of how Lange's exemplars of distinctively mathematical explanations — such as those involving mother's strawberries and the bridges of Konigsberg-fit within our incidentalist framework; nor is such a discussion necessary to raise our challenge that some explanations only satisfy CR-monism incidentally. 
number, $D(x)>0>D\left(x^{\prime}\right)$. Of course, had we begun with a point cooler than its antipode, then the signs would have changed: $D(x)<0<D\left(x^{\prime}\right)$. So, we can say quite generally that for any point, $x$ and its antipode $x^{\prime}, 0$ is between $D(x)$ and $D\left(x^{\prime}\right)$. The intermediate value theorem then entails that it is mathematically necessary that there must be some point $c$ where $D(c)=0$. But this would be a point that has the same temperature as its antipode.

Because the explanandum is shown to be mathematically necessary, the derivation from the intermediate value theorem fulfills the explanatory function under discussion. Furthermore, satisfaction of the veridicality and implication conditions, plus the fact that the explanans contains a mathematically necessary truth (the intermediate value theorem), suffice to realize this function. Hence, one can show that an explanandum has 'high-grade' (in this case, mathematical) necessity without deploying the dependency condition. So, this example provides prima facie evidence for counterfactual incidentalism.

CR-monists may reply to this challenge in several ways. First, they may deny our claim that this derivation fulfills its explanatory function independently of the dependency condition. To do so, they would need to recast the intermediate value theorem as a change-relating generalization. This suggests the following 'equatorial counterfactual' fills out the dependency condition:

EC: The intermediate value theorem supports at least one counterfactual of the following form: had $D(x)$ been discontinuous, then it would have been mathematically contingent (rather than mathematically necessary) that equatorial antipodes with identical temperatures exist.

This counterfactual raises several problems. For its antecedent to be true, the combination of Fourier's law and the law of energy conservation would have to be violated. In other words, it is physically impossible for temperature to be discontinuous. According to the standard Lewis-Stalnaker account, a counterfactual conditional is true in a world $w$ just in case, among all worlds in which its antecedent is true, the worlds in which its consequent is true are more similar to $w$ than the worlds in which its consequent is false. Since the antecedent is physically impossible, we need to evaluate the consequent in physically impossible worlds where temperature is discontinuous. However, the resulting evaluation depends crucially on our 
assumptions about modality. For instance, on the relatively uncontroversial assumptions that continuity, Fourier's law, or the law of energy conservation are essential to temperature and that 'temperature' is a rigid designator, the counterfactual is vacuously true. This plays right into our incidentalist gambit, for then the discontinuity of temperature could be replaced by any denial of essential properties (e.g. temperature is a sensation) to yield a vacuous counterfactual satisfying that condition. Similar problems arise for CR-monists if the laws of nature are regarded as metaphysically necessary.

Indeed, even without essentialist and necessitarian assumptions, evaluating EC faces serious challenges. Such evaluations require identifying worlds in which the physical laws fail to hold, but identical antipodal temperatures still exist. It is unclear, however, whether such worlds would even contain planetary objects resembling Earth, and hence whether equatorial antipodes would exist at all. Difficulties compound as to whether some of the worlds that are mathematically possible relative to these physically impossible worlds include identical antipodal temperatures. The latter holds only if the laws of physics obtain, which requires temperature to be continuous. So, it seems that the worlds mathematically accessible to discontinuous-temperature worlds in which antipodes exist will just be worlds in which temperature is continuous and the actual laws of mathematics hold. But in all those worlds, equitemperate antipodes will exist and EC will thereby be false. In other words, under the assumption that temperature is discontinuous and (yet) equitemperate equatorial antipodes exist, it is exceedingly difficult to see how the purely physical conditions needed to make their existence contingent would obtain without making temperature continuous. ${ }^{13}$

A second CR-monist response claims that the function $D(x)$ measuring the difference in antipodal temperatures, rather than the intermediate value theorem, supports change-relating counterfactuals. As a mathematical function, $D(x)$ does relate changes. However, the problem is that $D(x)$ does not relate changes between the explanans and the explanandum. In particular, changes in equatorial location $(x)$ will not explain why it is mathematically

${ }^{13} \mathrm{CR}$-monists might extract favorable evaluations of the counterfactuals by assuming that accessibility relations range over both possible and impossible worlds. However, this assumption raises significant conceptual and metaphysical challenges. See Nolan ([2013]). 
necessary that at least one equatorial point has the same temperature as its antipode. Thus, it will fail to realize the explanatory function of exhibiting high-grade necessity.

Third, CR-monists may deny any modal contrast in the explanandum and instead insist that the consequent of the counterfactual conditional is simply that no equatorial antipodes with identical temperatures exist. While the resulting counterfactual may be true, its truth-value no longer appears relevant to the explanation of high-grade necessity. Indeed, the discontinuity of temperature together with the intermediate value theorem does not imply anything about the existence of identical antipodal temperatures. This is a telltale sign that, once again, the intermediate value theorem is not a change-relating generalization. If temperature is no longer a continuous function, the intermediate value theorem becomes irrelevant to equatorial temperatures. Hence, it would seem that the CR-monist is either unable to identify a relevant counterfactual supported by the intermediate value theorem, or she is able to do so only by taking on the dialectical burden of adjudicating philosophical debates about the nature of modality.

Thus far, we have considered CR-monist objections that aim to show how the explanatory function of exhibiting high-grade necessities must make detours through the dependency condition. For the reasons presented, this seems like a dead end. Indeed, our responses to these objections suggest, pace Jansson and Saatsi ([2017]), that even the lower bar of establishing minimal CR-monism is in trouble. ${ }^{14}$ So, perhaps CR-monists' more promising route is to deny that the exhibition of high-grade necessity is a proper explanatory function.

To that end, CR-monists' fourth possible objection may involve denying that our derivation concerning equatorial antipodes is an explanation at all. However, it is hard to see how this can be done without begging the question. By theory-neutral criteria, the proof appears to be an explanation. For instance, in addition to satisfying the veridicality and implication conditions, it appears to be an acceptable answer to the explanation-seeking question, 'Why is

\footnotetext{
${ }^{14}$ Jansson and Saatsi ([2017]) do not consider the semantic problems about modality that we have raised here. Furthermore, they choose explanatory derivations that we think are easier to subsume under the CR-monist aegis because their minor premises are highly contingent. See our discussion of Craver and Povich ([2017]) for further discussion of this latter point.
} 
it mathematically necessary that there are equatorial antipodes with identical temperatures?' Furthermore, physical systems, such as equatorial temperatures, might reasonably be expected to exhibit nothing stronger than physical necessity, so the unexpected fact that some physical systems exhibit extra-physical necessity 'cries out' for explanation. Thus, asking the why-question seems well-motivated.

Finally, CR-monists may argue that if exhibiting high-grade necessity were an explanatory function, then all sorts of pseudo-explanations would be explanatory (Craver and Povich [2017]). For instance, basic trigonometry plus facts about the sun's angle of incidence and the shadow's length show that the tower's height is mathematically necessary. Yet, this is no explanation. Indeed, this might render certain intuitions underlying the previous objection into more theory-neutral criteria. The CR-monists' argument might be: because many things that confer high-grade necessity aren't explanations and this proof doesn't support change-relating counterfactuals, it is not an explanation in virtue of highlighting extra-physical necessities.

The first premise is already a nonstarter. Counterfactual incidentalists need not claim that everything that fulfills a given explanatory function is an explanation. Indeed, for certain pluralists, explanations will be so motley that the first premise of this argument is question-begging. And, of course, the second premise is question-begging for any critic of CR-monism. In addition to these argumentative flaws, the symmetry example is not on a par with the equatorial example for reasons readily captured by their differing capacities to reveal high-grade necessities, and that are independent of any change-relating counterfactuals that they support. In the symmetry example, the minor premise-concerning the sun's angle of incidence and the shadow's length-is modally weak; it is physically contingent. By contrast, in the equatorial example, the minor premise - that temperature is a continuous function-is physically necessary. Because of this difference, the equatorial example is clearly better suited to exhibit high-grade necessity. ${ }^{15}$ Hence, this objection does not undercut our case for counterfactual incidentalism.

\footnotetext{
${ }^{15}$ For similar reasons, the equatorial example cannot be 'reversed' into a non-explanatory modus tollens, as (Craver and Povich [2017]) do with Lange's ([2016]) examples of mathematical explanations.
} 
In summary, the equatorial explanation fulfills the explanatory function of showing the explanandum to partake of necessities stronger than the laws of nature. It does so without relying upon change-relating counterfactuals. Appealing to these counterfactuals is not only unnecessary for realizing this function, but leads to needless semantic puzzles about modality. Furthermore, we have seen that attempts to banish this function from the kingdom of explanatory ends is question-begging.

\subsection{Unity in diversity}

Another explanatory function is finding unity in apparent diversity. While this explanatory function is on full display in unificationist accounts of explanation-for example, Kitcher ([1989]) — it is also exhibited in recent discussions of renormalization group (RG) explanations. As above, we argue that counterfactual incidentalism shows how some RG explanations can execute this function, but not by virtue of supporting change-relating counterfactuals. We then anticipate and rebut several CR-monist objections.

RG explanations account for universal behavior across different systems. For instance, various fluids exhibit identical behavior near their respective critical points. Below the critical point, any fluid passing from a gas to a liquid at a fixed temperature due to an increase in pressure must pass through a state in which both gas and liquid are present. Above the critical point, this intermediary state does not occur. Similar critical behavior is found in ferromagnets, superfluid helium, and many other physical systems with starkly different microstructures. Strikingly, RG explanations account for why all of these heterogeneous systems behave identically during phase transitions near their critical points. Thus, it realizes the function of finding unity in apparent diversity.

Before proceeding, we distinguish two RG explananda. We call the first 'universality questions:'

Why do all of the different systems $S, S^{\prime}$, etc. exhibit the same behavior $B$ ?

In our example of phase transitions, this universality question is:

UQ: Why do all of these fluids, ferromagnets, etc. have order parameters proportional to $\epsilon^{\beta}$ near criticality? 
Here, an order parameter measures a system's distance from its critical point. For instance, in liquid-vapor phase transitions, the order parameter is:

$$
\Psi=\left|\rho_{l}-\rho_{v}\right|
$$

where $\rho_{l}$ is the density of a given fluid in its liquid phase and $\rho_{v}$ is the density of the fluid's corresponding vapor phase. Then the critical exponent $\beta$ describes the shape of the coexistence curve for a given fluid as follows:

$$
\Psi \propto \epsilon^{\beta}
$$

where $\epsilon=\left|\left(T_{c}-T\right) / T\right|$, which measures how close a system is to its critical temperature $T_{c}$.

Contrast universality questions with a second kind of RG explanandum that we call stability questions:

Why do the different systems $S, S^{\prime}$, etc. that exhibit the same behavior $B$ all have common feature $C$ ?

In the case of phase transitions, the stability question is:

SQ: Why do fluids, ferromagnets, etc. with order parameters proportional to $\epsilon^{\beta}$ near criticality all have dimensionality $d=3$ and symmetries that are broken upon passing through $T_{c}$ ?

Both UQ and SQ call for answers that realize the explanatory function of providing unity in apparent diversity. So, if either one succumbs to counterfactual incidentalism, then we have met our dialectical objective of challenging CR-monists. ${ }^{16}$

\footnotetext{
${ }^{16}$ Despite having many affinities with our view, Batterman and Rice ([2014], pp. 361) and Batterman ([2017]) seem to assume that an answer to UQ must also answer a host of other why-questions, including SQ. Quite reasonably, their critics have challenged this assumption Lange [2015]; Povich [2018]. Our argument does not invite this criticism, for even if answers to UQ support change-relating counterfactuals, there are answers to SQ for which kindred
} 
To that end, we focus on SQ. In our estimate, RG explanations of SQ rest on the following 'stability-based' reasoning:

(SB1) Among the systems with order parameters proportional to $\epsilon^{\beta}$ near criticality, no differences in the systems' micro-details lead to them having different dimensionalities and symmetries.

(SB2) Any possible differences in the systems' dimensionalities and symmetries would have been from differences in their micro-details.

(SB3) $\therefore$ All the systems with order parameters proportional to $\epsilon^{\beta}$ have the same dimensionality and symmetry properties.

RG methods furnish the first of these statements. More precisely, RG methods show that different systems' critical exponents remain unchanged under different $\lambda$-transformations. ${ }^{17}$ Variation in $\lambda$ is a microstructural change that, as Kadanoff ([1971], pp. 105) puts it, it 'takes us from the Ising model to the Heisenberg model [different models of magnetic behavior], or from $\mathrm{Ni}$ to $\mathrm{Fe}$, or from a nearest neighbor interaction to a next nearest neighbor interaction.' RG methods show how these $\lambda$-transformations make no difference to the universal behavior described by SQ. The resulting explanation (and SB1 in particular) thereby supports the following 'stability-exhibiting counterfactual:'

SEC: For all $\lambda$ and all $H_{0}$ and $H_{0}^{*}$ with order parameters proportional to $\epsilon^{\beta}$ near criticality, had a change in $\lambda$ changed $H_{0}$ to $H_{0}^{*}$, then $H_{0}$ and $H_{0}^{*}$ would both still have dimensionality $d=3$ and symmetries that are broken upon passing through $T_{c}$.

Here, $H_{0}$ and $H_{0}^{*}$ are two Hamiltonians, each characterizing interactions between a different system's components near criticality. For instance, $H_{0}$ might be the Hamiltonian representing interactions within one fluid, while $H_{0}^{*}$ represents the interactions within another fluid. counterfactuals are incidental. Additionally, Batterman and Rice's chief target are 'common features' approaches to RG and other 'minimal model' explanations (see Footnote 18); ours is CR-monism.

${ }^{17}$ See Batterman ([2017]) for further discussion. 
Moreover, SECs stand nearly diametrically opposed to the change-relating counterfactuals required to satisfy the dependency condition:

CR1: For all $\lambda$ and all $H_{0}$ and $H_{0}^{*}$ with order parameters proportional to $\epsilon^{\beta}$ near criticality, had a change in $\lambda$ changed $H_{0}$ to $H_{0}^{*}$, then $H_{0}$ and $H_{0}^{*}$ would not both still have dimensionality $d=3$ and symmetries that are broken upon passing through $T_{c}$.

Crucially, RG methods only need to underwrite stability-exhibiting counterfactuals to perform their function of answering stability questions. Since stability-exhibiting counterfactuals are near-opposites of change-relating counterfactuals such as CR1, we have another prima facie argument for counterfactual incidentalism.

Before defending this claim, let us briefly discuss how RG methods support SEC. Let $H_{0}$ be the Hamiltonian of a system $S$ going through a phase transition. Assume that $H_{0}$ represents the interactions between $S$ 's components in a fairly egalitarian manner, as is the case when $H_{0}$ is a highly detailed and accurate mapping of those interactions. However, only some of these interactions are of explanatory interest—namely what $S$ has in common with other systems during phase transitions near their critical points. To focus on just these features, scientists deploy the RG transformation: a 'coarse-graining' procedure that groups different degrees of freedom into larger units. Specifically, this transformation maps the physical Hamiltonian, which describes $S$, to a simpler Hamiltonian in the space of possible Hamiltonians. This allows scientists to identify the features that become more or less important at different scales, by preserving the aspects of the original Hamiltonian relevant to phase transitions while decreasing the degree of freedom with which components in the system interact. Scientists can thereby 'zoom out' on the Hamiltonian, and then rescale (renormalize) the components to resemble the original Hamiltonian.

Thus, the transformation on $H_{0}$ yields a simpler Hamiltonian $H_{1}$ which still resembles $S$, but describes fewer interactions. By repeating this process on $H_{1}, H_{2}$, etc., scientists eventually come to a 'fixed point' Hamiltonian, which excludes details of $S$ irrelevant to its phase transformation and makes the behavior of $S$ more comprehensible. $\lambda$-transformations are essentially differences in these irrelevant details. Thus, they 'drop out' in the system's flow to the fixed point. Renormalization thereby explains similar behavior across different systems 
because multiple physical systems - in this case, different fluids, ferromagnets, etc.-all flow to the same fixed point under repeated applications of renormalization techniques. This gives SB1 and SEC their generality.

With this account of RG explanations in hand, let us turn to some possible CR-monist objections. As before, their options are twofold: they must either insist that the explanatory function of finding unity in apparent diversity works by virtue of change-relating counterfactuals, or deny that it is an explanatory function at all.

Consider the first strategy. Some have argued that RG explanations traffic in change-relating counterfactual dependence relations (Povich [2018]; Reutlinger [2016]). We offer three replies. First and most importantly, these arguments at best only show that CR-monism can provide RG-inflected answers to universality questions (UQ); but not to stability questions (SQ). This does nothing to undermine our argument for counterfactual incidentalism, since that claim is existential, not universal: some explanations (namely RG explanations of SQ) do not work by virtue of supporting change-relating counterfactuals.

Second, CR-monists' treatment of the universality question imperils their prospects of adequately tackling the stability question. Observe that UQ's answer supports the following change-relating counterfactual:

CR2: Had one of the systems lacked common feature $C$ that it and all of these other fluids, ferromagnets, etc. actually share, then the order parameter would not be proportional to $\epsilon^{\beta}$ near criticality for all of these fluids, ferromagnets, etc. ${ }^{18}$

In the particular case of phase transitions, the common features are the symmetry of the order

\footnotetext{
${ }^{18} \mathrm{All}$ parties discussing $\mathrm{RG}$ explanations agree that even if there is a CR-explanation for each system S's critical behavior that appeals to S's microscopic idiosyncrasies, the aggregate of these micro-explanations does not capture RG explanations' core function of uncovering unity in apparent diversity. Hence, it would appear that counterfactual incidentalism is avoided only if RG explanations support counterfactuals such as CR2. This means that CR-monists must pursue what is sometimes called the 'commonality strategy.' However, the converse does not hold, as some commonality strategists, such as Lange ([2015]), are agnostic about RG explanations' compatibility with CR-monism.
} 
parameter and the spatial dimensionality of the various systems in the universality class. But, of course, SQ presupposes that the systems in question have these common features. On pain of circularity, explananda must not presuppose their explanantia, so CR-monists will have to find some other common feature to answer SQ. However, common features are few and far between in explanations of universal behaviors. Hence, CR-monists face a sizable burden of proof.

Third, it is not even clear that CR-monists' treatment of the universality question (much less the stability question) is immune to charges of incidentalism. The symmetry of the order parameter and the spatial dimensionality of the various systems are necessary features for all systems that exhibit the same critical behavior. So, they support change-relating counterfactuals such as CR2. However, many necessary conditions for an explanandum support incidental change-relating counterfactuals. For instance, Priscilla's being born is necessary for her buying a cup of coffee, but the following is incidental to the explanation of that purchase:

CR3: Had Priscilla not been born, she would not have bought a cup of coffee.

Arguably, the same worries arise with CR-monists' appeal to symmetry and dimensionality in their answer to UQ. For instance, in discussing RG explanations, Reutlinger ([2017b]) writes:

[...] the relevant symmetry properties and the spatial dimensionality are a part of an explanation of the multiply realized macroscopic behavior in question. The fact that microscopically very different physical systems have these properties in common (partially) explains why these physical systems display the same macro-behavior. (Reutlinger [2017b], pp.148)

Yet, rather strikingly, Reutlinger's ([2017b], pp. 146-147) discussion of RG explanations includes no reference whatsoever to these symmetry properties and dimensionalities-much in the same way that an explanation of Priscilla's coffee purchase in terms of her desire to wake up would not refer to her birth. To our knowledge, other CR-monists who claim that RG explanations work by supporting counterfactuals such as CR2 face similar worries.

Thus, CR-monists face steep challenges in showing that change-relating counterfactuals are always non-incidental to the explanatory function of finding unity in apparent diversity. So, perhaps CR-monists would be better served by denying that this is an explanatory function. 
One version of this objection would show that UQ, but not SQ, is a proper explanation-seeking question. However, it appears that scientists take SQ to be a legitimate why-question. Consider this quote from physicist Fisher ([1983]):

For instance, if it is asserted that the exponent [...] depends on the dimensionality, $d$, and on the symmetry number, $n$, but on no other factors, then the theorist's job is to explain why this is so and subject to what provisos. ${ }^{19}$ (Fisher [1983], pp. 47)

So, this CR-monist objection appears at odds with scientific practice.

However, perhaps CR-monists can grant the stability question is legitimate, but deny that its answer is fulfilling the explanatory function of finding unity in apparent diversity. To that end, they might argue that simply using the RG method to reveal the relevant unity is not explanatory unto itself (Povich [2018]). As noted above, RG methods only show that $\lambda$-transformations do not affect dimensionality and symmetry (SB1). However, a further assumption is that only these transformations could have affected these common features (SB2), and CR-monists have not shown that this second assumption is either illicit or capable of supporting non-incidental change-relating counterfactuals. Hence, the objection misplaces the burdens of proof. Similarly, some worry that applications of RG methods that simply revealed unity in apparent diversity would fall prey to symmetry problems (Lange [2015], pp. 295-298). However, it is unclear how this objection applies to our stability-based gloss of RG explanations. After all, one cannot swap out the conclusion (SB3) for either premise (SB1 or SB2) and preserve the validity of the inference. So, concerns about symmetry problems also appear misplaced.

In summary, we have argued that RG explanations' chief function, finding unity in apparent diversity, can be realized in ways that support counterfactual incidentalism. In particular, it appears that this function can be realized using stability-exhibiting counterfactuals, which are near-opposites of change-relating counterfactuals. Furthermore, CR-monist objections to these claims were found wanting.

\footnotetext{
${ }^{19}$ We learned of this quote from Batterman ([2017]), though he uses it to slightly different ends.
} 


\section{Conclusion}

We have argued that CR-monism—understood as the conjunction of the veridicality, implication, and dependency conditions_-does not provide sufficient conditions for explanations and, at best, only provides necessary conditions that are incidental to some explanations. We argued against the sufficiency of these conditions by showing that, by themselves, these three conditions did not distinguish explanations from pseudo-explanations, and that strengthening the conditions is most easily done by adopting a pluralistic stance. We then argued for the incidental nature of change-relating counterfactuals to some explanations by showing that these explanations fulfill their primary functions without relying on change-relating counterfactuals. In doing so, we also have defended a plurality of explanatory functions and a plurality of modal claims that underwrite these functions. Hence, pluralism appears to be the more plausible doctrine.

However, we see this less as a refutation of CR-monism, and more as an invitation for further discussion. Perhaps CR-monists can overcome the objections provided here. On this front, we hope that the two kinds of CR-monists glossed in Section 3-and perhaps other CR-monists—develop their conceptual resources in greater detail. Similarly, we have introduced the notion of an explanatory function in Section 4, but perhaps further analysis of this concept will yield different conclusions about CR-monism. Finally, perhaps it would be more fruitful to recast monism by appealing neither to necessary nor to sufficient conditions.

Similarly, we think that pluralists can continue to press their case in new directions. In addition to refining the framework offered here, we note that our target has only been the dependency condition. However, we also noted that a broader notion of representational success ought to replace the veridicality condition. To put this in slightly more provocative terms, explanations may map onto the world in a plurality of ways (Rohwer and Rice [2016]). Similarly, one may wonder if the implication condition, with its Hempelian stigmata, is sometimes false or incidental to scientific explanations.

What should also be clear is that the philosophy of science ought to 'move beyond causes' as Rice ([2015]) so pithily put it. Proper analyses of explanations now seem to be moving apace with a new suite of concepts: representation, modality, and explanatory function. 
Causal concepts appear derivative by comparison.

\title{
Funding
}

Khalifa acknowledges the University of Pittsburgh's Center for Philosophy of Science for its support during part of the writing of this manuscript; Doble, the same for Middlebury College's Undergraduate Collaborative Research Fund.

\section{Acknowledgements}

We would like to thank Chris Pincock, Collin Rice, Juha Saatsi, and three anonymous referees for comments on earlier drafts of this paper.

\author{
Kareem Khalifa \\ Middlebury College \\ Department of Philosophy \\ Twilight Hall 303A \\ Middlebury, VT 05753 USA \\ kkhalifa@middlebury.edu
}

Gabriel Doble

Harvard University

Law School

1563 Massachusetts Avenue

Cambridge, MA 02138

gdoble@jd20.law.harvard.edu

Jared Millson

Agnes Scott College

Philosophy Department 


\section{References}

Batterman, R. [2017]: 'Universality and RG Explanations', http://philsci-archive.pitt.edu/13460/, accessed 20 September 2017.

Batterman, R. W. and Rice, C. C. [2014]: 'Minimal Model Explanations', Philosophy of Science, 81(3), pp. 349-376.

Bokulich, A. [2011]: 'How Scientific Models Can Explain', Synthese, 180(1), pp. 33-45.

Cartwright, N. [1989]: Nature's Capacities and Their Measurement, Oxford University Press.

Craver, C. F. and Bechtel, W. [2007]: 'Top-Down Causation Without Top-Down Causes', Biology and Philosophy, 22(4), pp. 547-563.

Craver, C. F. and Povich, M. [2017]: 'The Directionality of Distinctively Mathematical Explanations', Studies in History and Philosophy of Science Part A, 63, pp. 31-38.

Fisher, M. E. [1983]: 'Scaling, Universality and Renormalization Group Theory’, in F. Hahne (ed.), Critical Phenomena, Berlin: Springer, pp. 18-29.

French, S. and Saatsi, J. [2018]: 'Symmetries and Explanatory Dependencies in Physics', in A. Reutlinger and J. Saatsi (eds), Explanation Beyond Causation: Philosophical Perspectives on Non-Causal Explanations, Oxford: Oxford University Press, pp. 185-205.

Hochstein, E. [2017]: 'Why One Model is Never Enough: A Defense of Explanatory Holism', Biology and Philosophy, 32(6), pp. 1105-1125.

Jansson, L. and Saatsi, J. [2017]: 'Explanatory Abstractions', The British Journal for the Philosophy of Science, pp. axx016. <http://dx.doi.org/10.1093/bjps/axx016>.

Kadanoff, L. P. [1971]: 'Critical Behavior: Universality and Scaling', in M. Green (ed.), International School of Physics 'Enrico Fermi' Course, Italian Physical Society, Academic Press, pp. 100-117. 
Kitcher, P. [1989]: 'Explanatory Unification and the Causal Structure of the World', in P. Kitcher and W. Salmon (eds), Scientific Explanation, Minneapolis: University of Minnesota Press, pp. 410-505.

Kostić, D. [2016]: 'Mechanistic and Topological Explanations: An Introduction', Synthese, 195(1).

Lange, M. [2015]: 'On “Minimal Model Explanations”: A Reply to Batterman and Rice', Philosophy of Science, 82(2), pp. 292-305.

Lange, M. [2016]: Because Without Cause: Non-Causal Explanations in Science and Mathematics, New York: Oxford University Press.

Nolan, D. P. [2013]: 'Impossible Worlds', Philosophy Compass, 8(4), pp. 360-372.

Pexton, M. [2014]: 'How Dimensional Analysis Can Explain', Synthese, 191(10), pp. 2333-2351.

Pincock, C. [Forthcoming]: ‘Accommodating Explanatory Pluralism', in A. Reutlinger and J. Saatsi (eds), Explanation Beyond Causation : Philosophical Perspectives on Non-causal Explanations, New York: Oxford University Press.

Povich, M. [2018]: 'Minimal Models and the Generalized Ontic Conception of Scientific Explanation', British Journal for the Philosophy of Science, 69(1), pp. 117-137.

Reutlinger, A. [2016]: 'Is There A Monist Theory of Causal and Noncausal Explanations? The Counterfactual Theory of Scientific Explanation', Philosophy of Science, 83(5), pp. 733-745.

Reutlinger, A. [2017a]: 'Do Renormalization Group Explanations Conform to the Commonality Strategy?', Journal for General Philosophy of Science / Zeitschrift für Allgemeine Wissenschaftstheorie, 48(1), pp. 143-150.

Reutlinger, A. [2017b]: 'Explanation Beyond Causation? New Directions in the Philosophy of Scientific Explanation', Philosophy Compass, 12(2), pp. e12395. 
Rice, C. [2015]: 'Moving Beyond Causes: Optimality Models and Scientific Explanation', Noûs, 49(3), pp. 589-615.

Rohwer, Y. and Rice, C. [2016]: 'How Are Models and Explanations Related?', Erkenntnis, 81(5), pp. 1127-1148.

Saatsi, J. [2018]: ‘On Explanations From Geometry of Motion', British Journal for the Philosophy of Science, 69(1), pp. 253-273.

Saatsi, J. and Pexton, M. [2013]: 'Reassessing Woodward's Account of Explanation: Regularities, Counterfactuals, and Noncausal Explanations', Philosophy of Science, 80(5), pp. 613-624.

Salmon, W. C. [1984]: Scientific Explanation and the Causal Structure of the World, Princeton: Princeton University Press.

Skow, B. [2013]: ‘Are There Non-Causal Explanations (of Particular Events)?', British Journal for the Philosophy of Science, (3), pp. axs047.

Strevens, M. [2008]: Depth: An Account of Scientific Explanation, Cambridge: Harvard University Press.

Woodward, J. [2003]: Making Things Happen: A Theory of Causal Explanation, New York: Oxford University Press.

Ylikoski, P. [2013]: 'Causal and Constitutive Explanation Compared', Erkenntnis, 78(2), pp. $277-297$.

Ylikoski, P. [2014]: 'Rethinking Micro-Macro Relations', in Rethinking the Individualism-Holism Debate, Springer, pp. 117-135. 